\title{
Journal of Biomedicine
}

Review

\section{N-Glycosylation of IgG Immunoglobulin and its clinical significance}

\author{
Maria Papakonstantinou $1^{*}$, Georgios Dryllis ${ }^{2 *}$, Maria Efstathopoulou ${ }^{2}$, Dimitra Vlachopanou ${ }^{1}$, Michalis \\ Kechriotis ${ }^{1}$, Serena Valsami2 ${ }^{\bowtie}$ \\ 1. Blood Transfusion Service, General Hospital of Nikea, Andrea Petrou Mantouvalou, 184 54, Athens, Greece \\ 2. Hematology Laboratory-Blood Bank, ARETAEIO Hospital, National and Kapodistrian University of Athens 76 Vasilissis Sofias Ave., 115 28, Athens, \\ Greece \\ *These authors contributed equally.
}

$\square$ Corresponding author: Serena Valsami, Assistant Professor, Medical School of the University of Athens, Hematology Laboratory-Blood Bank, ARETAEIO Hospital, National and Kapodistrian University of Athens 76 Vasilissis Sofias Ave., 115 28, Athens, Greece. Tel.: +30 210-7286238; Fax: +30 210-7230785; Email: gdrillis@yahoo.gr

(c) The author(s). This is an open access article distributed under the terms of the Creative Commons Attribution License (https://creativecommons.org/licenses/by/4.0/). See http://ivyspring.com/terms for full terms and conditions.

Received: 2019.02.08; Accepted: 2019.10.11; Published: 2019.10.13

\begin{abstract}
The IgG immunoglobulins are the main immunoglobulins in human beings. They have the longest half time and are the most studied of all. After the $\mathrm{Ag}$ binding, there is a signaling through the $\mathrm{Fc}$ region.

The post translational modification of the immunoglobulin mainly includes the N-Glycosylation and mostly the $\lg G$ one. The $\lg G$ s represent the antibodies, which are the mediators of the immunity, against extracellular bacteria and toxins.

The Fcy receptors exist in all hemopeitic cells. The extracellular parts of the receptors show high grade of homology in their amino acid sequences. Monoclonal antibodies can distinguish them.

In addition to nucleic acids, proteins and lipids, sugars are also fundamental components of animal systems. Compared with advances in genomics and proteomics, the study of glycoscience is under investigation.

Changes in glycosylation may be used as biological markers for screening in relation to healthy subjects. It may help in the discovery of patients in early stages of the disease and thus be used as a biological marker to select patients with better prognosis and therefore to use as chemotherapy chemotherapeutic agents with lower toxicity.
\end{abstract}

Key words: N-Glycosylation, Immunoglobulin

\section{Introduction}

The post translational modification of the immunoglobulin mainly includes the N-Glycosylation and mostly the IgG one. The IgGs represent the antibodies, which are the mediators of the immunity, against extracellular bacteria and toxins.

The N-Glycosylation takes place in the rough endoplasmic reticulum and the Golgi apparatus. It is about adding an oligosaccharide in the amide nitrogen atom Nof asparagine Asn-297, which is an aminoacid residue placed in 297 positions in the Fc region of the immunoglobulin. The oligosaccharide is comprised of the core heptasaccharide which is the GlcNAc-GlcNAc-mannose-(mannose)2-(GlcNAc)2, branched in two arms, expanding from the constant core structure of mannose-(GlcNAc)2, by adding a mannose and a GlcNAc in each arm. Subsequently a galactose or a sialic acid can be added in each arm.

The N-Glycosylation influences the structure of the IgG immunoglobulin and finally effects the interaction of the IgG antibodies with the $\mathrm{Fcy}$ receptors $(\mathrm{Fc} \gamma \mathrm{Rs})$ of the immunity cells, reflecting a 
possible pathogenic role in several diseases, such as autoimmune disorders and malignancies.

\section{IgG IMMUNOGLOBULINS}

\subsection{The IgG Immunoglobulins -Structure}

The IgG immunoglobulins are the main immunoglobulins in human beings. They have the longest half time and are the most studied of all.

The IgG immunoglobulins ( $150 \mathrm{kDa})$ consist of 2 identical light ( $\mathrm{\kappa}$ and $\lambda$ ) and two heavy chains, which bind with each other (every light chain with one heavy and every heavy with the other heavy chain). The chains are binded with covalent, disulfide bonds. The light chains are identical $(23 \mathrm{kDa})$ as well as the heavy chains $(50 \mathrm{kDa})$.

Using a proteolytic enzyme, the papaine, the peptidic bonds of the IgG break, resulting in 3 fragments, two Fabs (Fragments of antigen binding) and an Fc (Fragment crystallizable). Every Fab part consists of the whole light chain and part from the heavy chain. The configuration of the 2 Fabs makes the IgG molecule look like a " $Y$ ".

The light chains $\mathrm{L}$ of the IgG are consisted of 2 parts, the variable domain VL and the constant domain $\mathrm{C}$, while the heavy chains of 4 parts, the $\mathrm{VH}$, $\mathrm{CH} 1, \mathrm{CH} 2, \mathrm{CH} 3$, starting from the N-terminal of the chains (Figure 1).

\section{a) Four chain structure of IgG}
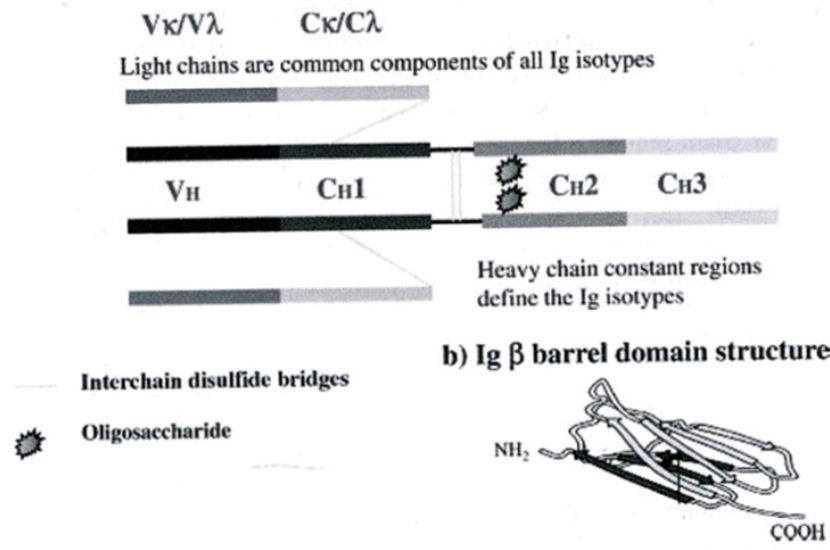

Figure 1: Walsh G. "Post-translation Modification of Protein Biopharmaceuticals. Part One, Glycosylation"

Each light chain is composed from a big domain, which is constant in different types of antibodies (even in different species). The constant region has an almost identical sequence of aminoacids in the C-terminal, while the $\mathrm{N}$ - terminal shows great diversity, regarding the aminoacid sequence and it is called Variable region. (1) (2) (7)

The constant region has an almost identical amino acid sequence to the C-terminus while at the
N-terminal it exhibits great variability in the variable region. Largest diversity extends to light chains in regions called Hypervariable regions or Complementarity Determining Regions CDR. Each CDR consists of 10 amino acid residues designated CDR1, CDR2, CDR3 (7)

The $\mathrm{Ab}$ has high segment flexibility. The two Fab parts move regarding one another and the Fc part, while binding to the Ag. The domain that gives that flexibility is called hinge region, it is important for the function of the IgGs and it is placed between the $\mathrm{CH} 1$ and $\mathrm{CH} 2$. On the Fab region is formed the flat surface for Abs binding or special cavities for haptens binding. (3)

\subsection{The IgG Immunoglobulins-Function}

After the $\mathrm{Ag}$ binding, there is a signaling through the $\mathrm{Fc}$ region. The $\operatorname{IgG}$ molecules have 2 kinds of function:

1) They form special immune complexes with Ag that bind the Fab region.

2) Functions through the Fc region (activation of the complement cascade or cell activation through Fc receptors).

Antibody Dependent Cellular Cytotoxicity (ADCC): The NKs take action,binding to cells covered. This is achieved though the FcyRIII (CD16) receptor, which binds to the Fc region of the IgG, attached to the target cell. The signal produced by the $\mathrm{Fc} / \mathrm{Fc}_{\mathrm{C}} \mathrm{RIII}$ connection activate the NK cells, which release their granules and destroy the IgG covered targeted cells. $(4,5)$.

\section{Activation of the Complement Cascade}

There are 3 ways of complement activation, the alternative, the classical and the pathway of lectin. The alternative and the lectin pathway belong to the innate immunity, whereas the classical belongs to the acquired immunity. The above end up to the complement dependent cytotoxicity (CDC).

In the alternative pathway, the $\mathrm{C} 3 \mathrm{~b}$ part of the C3 fragment of the complement lies on the microbe membrane and the complement cascade starts.

In the classical pathway the $\operatorname{IgM}$ immunoglobulin or the IgG1 and IgG3 subclasses of IgG bind to the Ag on the microbes' surface. The Fc parts of the antibodies come closer and two neighbor Fcs bind to the $\mathrm{C} 1$ fragment of the complement. Subsequently the complement cascade is activated.

In the lectin pathway, a serum lectin, the Mannose Binding lectin (MBL) binds to the mannose of the microbes and then the complement cascade is activated.

In the final steps of the cascade, in all three pathways, the Membrane Attack Complex (MAC) is 
formed, which in turn creates holes in the membrane of the target cell. Finally the cell is destroyed due to osmotic lysis. (6)

The IgG achieve its goal through the $\mathrm{Fc}_{\mathrm{c}}$ binding to the Fcy receptor that exists on operating cells, such as the macrophages.

\subsection{FcY RECEPTORS: STRUCTURE AND FUNCTION}

The Fcy receptors exist in all hemopeitic cells. The extracellular parts of the receptors show high grade of homology in their amino acid sequences. Monoclonal antibodies can distinguish them. The CD64 react to the FcyRI, the CD32 to the FcyRII and CD16 to the FcyRIII. All of them are transmembrane glycoproteins and belong to the superfamily of the immunoglobulins. They differ in the intracellular part,having tyrosine -based activating (ITAM) and tyrosine -based inhibitory motifs (ITIM). (7) Inflammatory cytokines regulate their expression.

The human IgG receptors include activating receptors (FcyRI, FcyRIIC,FcyRIIA and FcyRIIIA), an inhibitory receptor $(\mathrm{Fc} \gamma \mathrm{RIIB})$, a receptor of uncertain function (FcyRIIIB) and the $F_{c} \gamma R n$, which is involved in the IgG clearance and transfer. They form from an extracellular part with two or three domains (the immunoglobulin like domain), a transmembrane and an intracellular part.
Human IgG receptors consist of activating

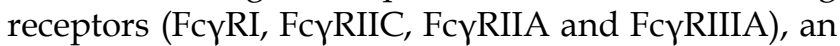
inhibitory receptor (FcyRIIB), a fuzzy function receptor $\left(\mathrm{F}_{\mathrm{c}} \mathrm{R} \mathrm{RIIIB}\right)$ and one $(\mathrm{Fc} \gamma \mathrm{Rn})$ involved in the clearance and transport of IgG, other functions. They consist of an extracellular domain with two or three domains, similar to IgG (immunoglobulin-like domain), a transmembrane and a cytoplasmic or intracellular part (Figure 2).

Following binding to polymeric agonists, $\mathrm{F}_{\mathrm{c}} \mathrm{R}$ activators accumulate in the cell membrane and carry intracellular signaling via the ITAM18 motif (immunoreceptor tyrosine-based activation motif). Especially for FcyRIIIA, the motif is found in the accompanying $\gamma$ chain. ITAM allows the recruitment of tyrosine kinases of the SRC and SYC family and this signaling pathway ultimately activates the cell, causing the production of cytokines and chemokines by the cell. (8)

The immunoreceptor tyrosine-based inhibition motif (ITIM) is located at the cytoplasmic domain of the FcyRIIB inhibitory receptor. After binding to its ligand, it recruits SRC tyrosine kinases and phosphotyrosine phosphatases are finally recruited that reduce cell activation by molecules involved in cell signaling. Using monoclonal $\mathrm{Ab}$, the following were found with respect to the cellular distribution of FcyRs immunoglobulin receptors presented in Table 1 below.

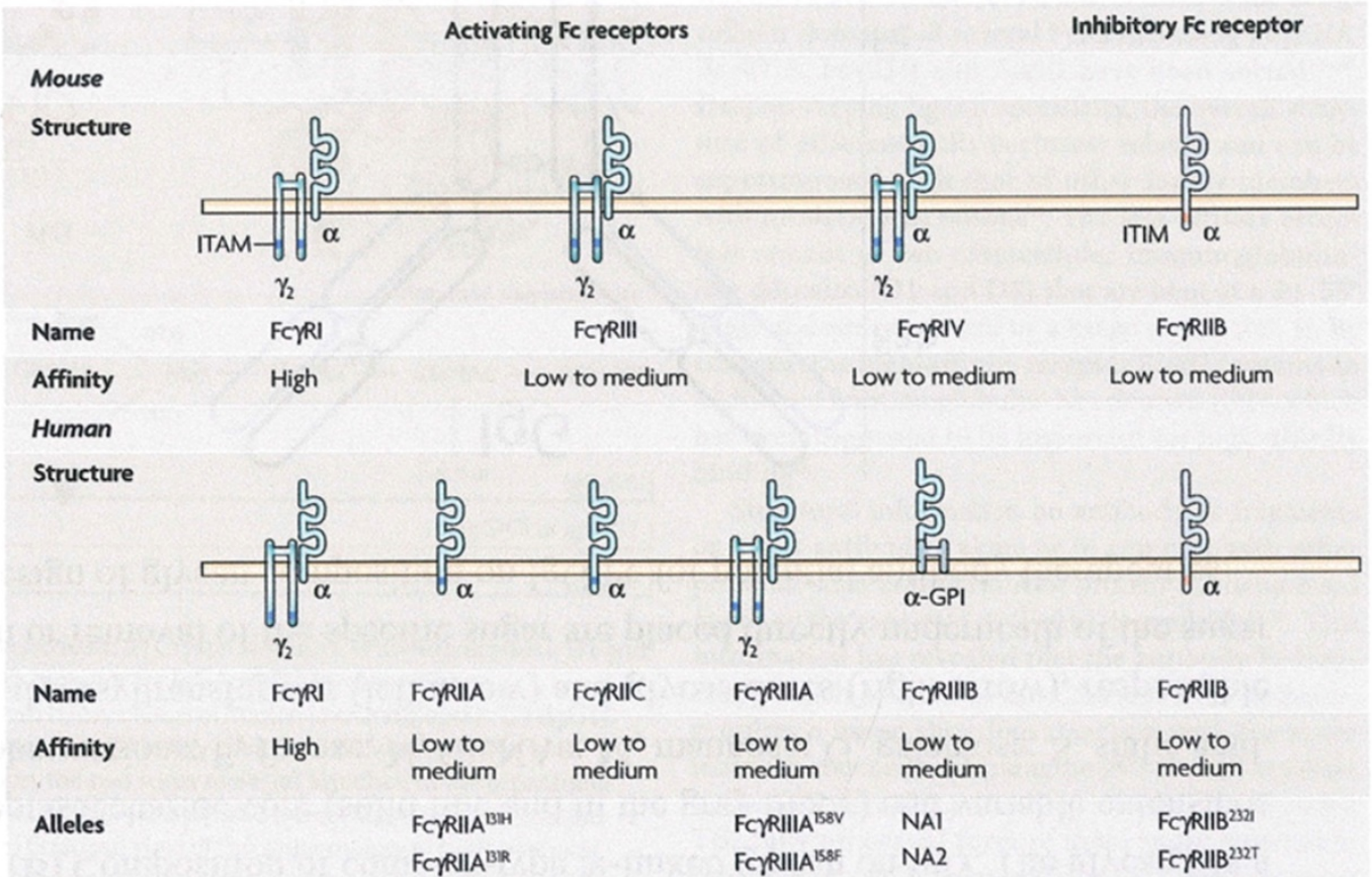

Figure 2: The family of Fc receptors from murine and human lgG. There is an affinity FcyRI receptor. All other receptors have a low average affinity for the Fc portion of the antibodies. FcyRIlb Inhibitory Receptor with a chain containing an Immunoreceptor Tyrosine Based Inhibitory Motif (ITIM) in the cytoplasmic segment. Activating receptors consist of an $\alpha$-chain linker and an $\gamma$-chain in the form of a messenger-containing dimer and contain Immunoreceptor Tyrosine Based Activator Motif (ITAM). (Fcy receptors as regulators of immune responses; by Falk Nimmerjahn et al.) 
Table 1: Distribution of FcyRs immunoglobulin receptors

\begin{tabular}{lll}
\hline Type & Cell distribution & Actions \\
\hline FcyRI(CD64) & $\begin{array}{l}\text { Monocytes / macrophages and } \\
\text { dendritic cells }\end{array}$ & $\begin{array}{l}\text { Phagocytosis } \\
\text { Cell Activation }\end{array}$ \\
$\begin{array}{l}\text { FcyIIA(CD32A) } \\
\text { FcyIIB(CD32B) }\end{array}$ & $\begin{array}{l}\text { Myeloid Cells } \\
\text { All immune cells, mainly in B-cells, } \\
\text { basophils and not in NK }\end{array}$ & $\begin{array}{l}\text { Phagocytosis } \\
\text { Inhibition of cell activity }\end{array}$ \\
FcyIIIA(CD16A) & Monocytes / macrophages, NK & $\begin{array}{l}\text { Induces ADCC and } \\
\text { cytokine release }\end{array}$ \\
FcyIIIB(CD16B) & Neutrophils & Microbial destruction
\end{tabular}

Activating receptors can be downregulated by the FcyRIIB inhibitory receptor only in cells expressing the inhibitory receptor (7). After recognition of $\mathrm{Ag}$ binding to $\mathrm{IgG}$, alteration of conformation, IgG shifting and $\mathrm{FC}_{\mathrm{C}}$ migration are made, allowing for interaction with FcR. In particular an asymmetric opening of about $7 \mathrm{~A} 0$ occurs between the $\mathrm{N}$-terminal ends of the $\mathrm{CH} 2$ domains. The interaction of the $F_{C}$ portion of IgG and FcR occurs with the $\mathrm{N}$-terminal of the $\mathrm{CH} 2$ segment within the low hinge region by combining hydrogen bonds, ionic bonds and hydrophobic interactions. FcRs which have been linked to the $F_{C}$ portion of IgG trigger phosphorylation of ITAMs by tyrosine kinases, members of the src kinase family, to achieve signal transduction. The result is phagocytosis, Ab-dependent cytotoxicity (ADCC) of Ab-coated target cells, as well as the release (by degranulation or directly) of proteolytic enzymes, oxygen free radicals and inflammatory mediators. Effector cells express 2 kinds of $\mathrm{F}_{\mathrm{C}} \mathrm{R}$ Rs, the activating and the inhibitory. The relationship of expression of these opposite systems determines the immune cell response.

Examples of immunoglobulin receptors and their role in vivo: Fcallb: inhibits inflammation. FcgIIIa: promotes ADCC. C1qR: initiation of the classical pathway in the complement cascade. FcRn: protects $\mathrm{Ab}$ from degradation by lysosomes to prolong its half-life (9).

The immune complexes also activate the complement by binding to the $\mathrm{Clq}$ fragment of the complement. The glycoform (carbohydrate composition) of the receptors may activate the complement via the lectin pathway. Depending on their type, glycoforms can be linked to mannose receptors expressed on antigen presenting cells. Since there is a defective configuration of the $\mathrm{Fc}_{\mathrm{c}}$ portion that bounds with glycans, the binding of C1q complement protein and $\mathrm{FCY}_{\mathrm{C}}$ receptors; disrupted. Th defective configuration is the result of the Fc deglycosylation.

FcnRs, (neonatal Fc receptors) concern another important biological activity of IgG, which is the transfer of maternal Abs the placenta to the fetus and through breast milk to the neonate (the placenta and the epithelial cells of the neonates' bowel express
FcRn receptors). Human embryos and neonates up to the age of one year do not have a fully developed immune system and their protection is taken up by the maternal Abs (passive immunity). On the other hand, these receptors also have the function of transporting the IgG antibody from the subsequent lysosomal degradation and keeping it in circulation. (10)

\section{PROTEIN GLUCOSYLATION}

\subsection{Introduction to post-translated modification of proteins}

In addition to nucleic acids, proteins and lipids, sugars are also fundamental components of animal systems. Compared with advances in genomics and proteomics, the study of glycoscience is under investigation. It is known that the flow of genetic information starts from DNA and ends up in proteins. After protein-synthesis, proteins interact with other proteins in order to form networks of interaction. However, in order a protein to be functional requires post-translational modification (PTM), which usually involves the addition of sugars to newly synthesized proteins. This is the case in the endoplasmicreticulum and Golgi apparatus. (11) Therefore, adding sugar to proteins is considered the last step in the flow of genetic information.

The cell synthesizes thousands of polysaccharides consisting of a limited number of monosaccharides. The structure of polysaccharides as well as their binding site to proteins has some information about the phenotype of the cell.

The most important post-translational modification as mentioned above is the glycosylation of proteins distinguished in $\mathrm{N}$-glycosylation and O-glycosylation.

In this work, the N-glycosylation of immunoglobulins, and in particular the most frequent immunoglobulin in human IgG (12), is being developed.

$\mathrm{N}$-linked glycosylation, which is the addition of an oligosaccharide to an amide nitrogen atom $(\mathrm{N})$, in the amino acid residue of Asparagine (Asn) in proteins, is important for the structure and function of proteins. That happens to the Eukaryotes, widely in ancient bacteria, rarely in eubacteria. The nature of glycosylation depends on the protein, the cell that is expressed and the type of organism. N-linked and O-linked glycosylation have been studied with respect to the locations that occur, the synthetic procedures and the enzymes that catalyze these reactions. We will discuss in more detail $\mathrm{N}$ glycosylation. Glycosylation process involves lipidlinked precursor oligosaccharides, monosaccharide 
residues, monosaccharide transfer nucleosides, receptor peptides, intermediates and end products that are glycoproteins. Enzymes such as glycosyltransferases (Gtfs) and glycosidases are involved.

The monosaccharides constituting the glycans of the human glycoproteins are as follows: Glc: D-glucose, Man: D-mannose, GlcNAc: N-acetyl-Dglucosamine, Gal: D-galactose, Fuc: L-fucoze GalNAc: N-acetyl-D galactosamine Xyl: D-xylose, NeuNAc: $\mathrm{N}$-acetylneuraminic acid or sialic acid. (13) The main synthetic procedures and addition and cutoff processes occur in the rough endoplasmic reticulum (RER) and the Golgi apparatus. The oligosaccharide addition is made to the amino acid residue of proteins (asparagine - Asn).

\section{2. N-glucosylation of IgG}

In general

Overall, the IgG molecule lacks of symmetry. The electron density maps show all the parts of the $\mathrm{Ab}$ and the hinge region and the adherent carbohydrates in each $\mathrm{CH} 2$ domain in the interface between each other, responsible for the weak bridge between them (14) (Figure 3).

Fab

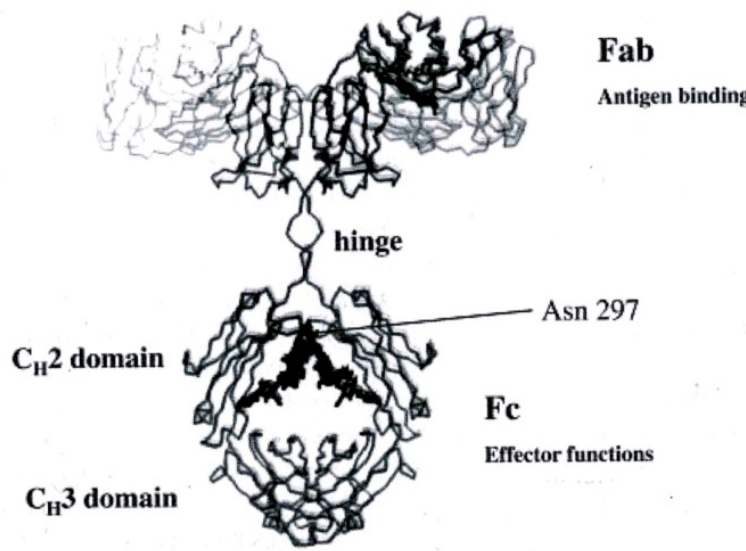

Figure 3: (Walsh, G., Post-translational Modification of Protein Biopharmaceuticals, Part One, Clycosylation)

Glycosylation suffer the Fc segments of IgG more stably, but also the Fab segments at conserved sites. The oligosaccharides are large in size (about $2 \mathrm{KDa}$ ) and, as they are not limited by their position, they are flexible. They have a crucial structural and functional role, such as lectine binding (Mannose Binding Lectin, MBL) due to the presence of glycan epitopes. They play a role in maintaining solubility, modulating the molecule, facilitating subcellular movement and clearance of the molecule. There is defective glycosylation (with a role in disease pathogenesis) in conditions such as rheumatoid arthritis and is applicable to the development of diagnostic biomarkers.

The processing of the oligosaccharides is specific for each cell and each individual protein while the three-dimensional structure of the protein molecule around the glycosylation site controls the processing of the glycan at each binding site. It seems that a large surface of each protein is covered by sugars due to their large size and flexibility. Thus, sugars can also block the interaction of $\mathrm{Ab}$ with their receptors.

The N-linked glycosylation site is located in the Asn-297 (15) (Figure 4) residue in the conserved sequence Asn-X-Ser / Thr (where X each amino acid except proline) to the amino-terminal region of the $\mathrm{CH} 2$ portion of IgG. The carbohydrate sequence attached to Asn-297 in the Fc portion of IgG is shown in Figure 4.

The prominent core structure of humans' saccharide is the octasaccharide FucGLcNAc2 Man3GLcNAc2 with Fuc (fucose) in the original GLcNAc linked to Asn-297. The structure is expanded by the addition of Gal (galactose) and sialic acid. The Man (1-6) branch, branched by Man4 is directed toward $\mathrm{CH} 2-\mathrm{CH} 3$ while the Man (1-3) arm extends into the gap between the $\mathrm{CH} 2$ portions and contacts the (1-3) arm of the opposite $\mathrm{CH}$-linked oligosaccharide. (Figure 4)

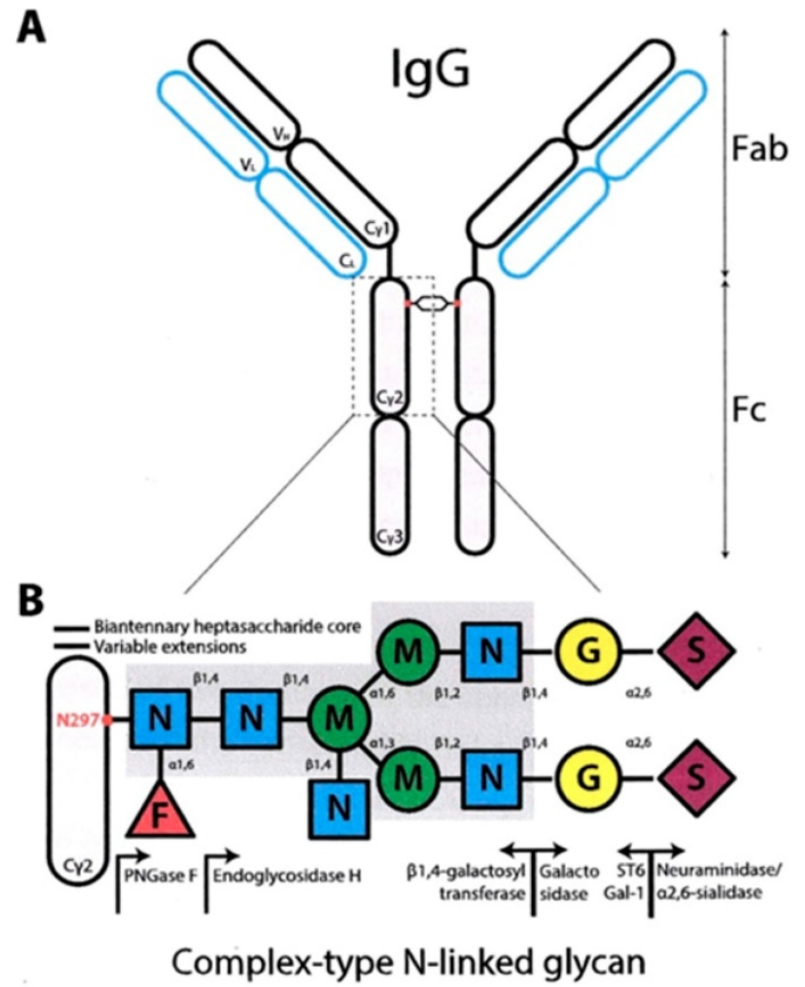

Figure 4: «Antibody Glycosylation and Inflammation” Kai-Ting C. Shade, et al. 


\subsection{The importance of Glucosylation in the structure of IgG Immunoglobins}

The two $\mathrm{CH} 2$ segments are bridged by the glycans, from one for each segment, occupying the inner interstitial space formed between the $\mathrm{CH} 2$ segments. Thus, a more open configuration is provided to the IgG Fc domain to permit binding to FcyRs. Non-covalent interactions are developed between the Fc portion of IgG and the glycans to form a stable conformation that affects the quaternary structure of the $\mathrm{Fc}_{\mathrm{C}}$ fragment. Studies with X-ray crystallography have shown that removal of glycan residues causes changes in the $\mathrm{CH} 2$ configuration due to loss of glycan-glycan and glycan-protein interaction. Each conserved N-linked glycosylation site (Asn-297) takes part in about 70 non-covalent interactions (16) with the protein surface that restrict the movement of the glycan. The Man (1-3) branch of the sugar arm does not come into contact with the protein surface and protrudes into the space within the Fc domain (14). The two $\mathrm{CH} 2$ portions of the Fc form an angle around the $\mathrm{CH} 2-\mathrm{CH} 3$ link. The distance between the two $\mathrm{CH} 2$ segments is regulated by the oligosaccharides attached there, in order to move close or away from one another. This bend determines the opening of the horseshoe IgG segment. The inverse relationship between the length of the oligosaccharide and the distance between the two $\mathrm{CH} 2$ regions of the $\mathrm{Fc}$ segment (as small as the length the greater the distance) is due to the loss of weak interactions that stabilize the contact between oligosaccharide-protein and rend the $\mathrm{CH} 2$ portion non deformable. (17) The presence of glycans is essential to maintain the Fc structure, which is crucial for $\mathrm{Ab}$ mediated effector functions. Changes in the composition of saccharide residues dramatically affect the activity of the antibody.

IgGs exhibit a distinct number of glycoforms that reflects the large variety in their oligosaccharide type. These conserved oligosaccharides have structural and functional roles that have been explored.

\subsection{Types of Glycoforms of IgG.}

The Fc glucan of IgG with its two arm structure (biantennary) have great heterogeneity depending on the presence or absence of various end-sugars. The presence of the two Fc-glycans linked to each of the two heavy chains (CH2) of the Ab at the Asn-297 site is critical for $\mathrm{Ab}$ activities in terms of effector functions, such as $\mathrm{Ab}$ dependent cytotoxicity (ADCC) and activation of the complement.

IgG glycoproteins consist of $82-96 \%$ of protein and $4-18 \%$ of carbohydrates. $(7,15)$

The glycosylation of antibody is a post-translational modification (PTM), and includes variation in the glycosylation site, the type, length and branching of the oligosaccharide arms. The oligosaccharides have heterogeneity. They have a complex structure and usually have two arms (biantennary). Each Asn-297 contains one of the family of a total of 32 types of glycans (Figure 5).

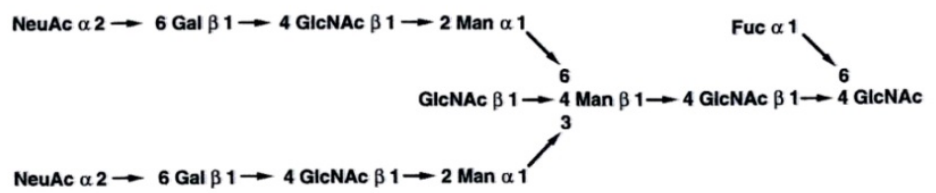

A
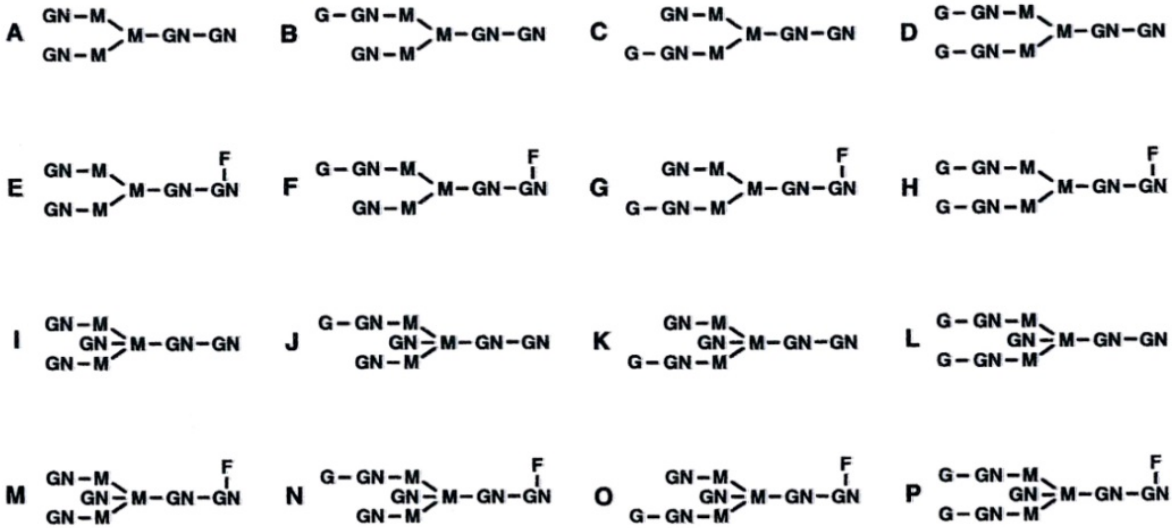

Figure 5: Structure of the fully matured complex oligosaccharide type dual arm (top) and 16 possible natural oligosaccharide structures contained in lgG-Fc (A-P) F, Fucose G, Galactose GN, N-Acetylglucosamine, M, Manoses, Carlos B. Hirschberg, Martin D. Snider). Potential forms of glycoforms, according to the current nomenclature system used are: G0: galactose-free hepta-saccharide, (G); G1: addition of a galactose (G) to one arm or another; $\mathbf{G 2 :}$ addition of two galactoses, (G), one in each arm; GoF: Fucose addition to the original GIcNAc, (GN); G1F, G2F: addition of fucose, (F) and galactose, $(\mathrm{G})$, according to the above; G0B G0F: addition of one more bisecting GlcNAc arm, (GN), to the original heptasaccharide; G1B, G2B.; G0BF, G1BF, G2BF. 
Note that there may be 2 G1 forms in each case, depending on which arm is added galactose, addition to the a (1-3) or a (1-6) arm. $(19,13)$

The type of IgG glycoform depends on species, age, pathological conditions, culture conditions for IgG-producing cells, pregnancy. (20) The glycan types in the Fc segment affect the intracellular pathway of each IgG molecule in its pathway of biosynthesis.

\subsection{Importance of Galactose}

There are 3 glycoform subfamilies of galactosylation that are IgG-G0 (no galactose) or IgG-G1 (with galactose in one arm) and IgG-G2 (galactose in both arms). They are found among 32 kinds of glycoforms in human serum. The distribution in normal human human serum is quantitatively $20-25 \%$ IgG-G0,35-45\% IgG-G1 and 10-20\% IgG-G2. (21)

Differences in galactosylation according to gender and age are observed. The female has a higher degree of galactosylation as well as sialylation compared to the male. Galactosylation decreases with age during adulthood. Overall galactosylation, however, remains stable in children and adolescents. Differences were observed in infants compared to children, with neonates having high galactosylation. Similarly, high levels of galactosylation also occur in pregnant women in the third trimester. The neonatal FcRn receptor mediates the transfer of IgG through the placenta to the fetus, resulting in the mother and neonate having the same glycosylation profile at birth. High galactosylation in pregnant women is an anti-inflammatory feature of IgG and contributes to suppressing the mother's immune response during pregnancy. Differences in galactosylation between children and infants (in children reduction of galactosylation in relation to neonates) reflect the fact that the infant receives antibodies from the mother during pregnancy while children produce their own antibodies. (22)

It is known that IgG interactions with $F_{c} \gamma$ Rs cell receptors depend on the presence of carbohydrates. Non-glycosylated IgG does not bind to receptors in monocytes, macrophages and B-cells. The specific Gal removal results in reduced affinity for the $F_{C Y}$ receptor. (23)

Regarding the effect of Gal on ADCC there are conflicting results in the studies. Others find no correlation, while others have found that the existence of Gal residues increases ADCC. (15)

It has been observed that in healthy subjects $25 \%$ is IgG-G0 type, whereas in rheumatoid arthritis, systemic lupus erythematosus, Crohn's disease and other autoimmune diseases, it accounts for $50 \%$ of serum IgG. The absence of Gal in the high man hepta-saccharide leaves man (mannose) exposed to the mannose-binding lectin (MBL) involved in complement-dependent cytotoxicity (CDC). MBL is the first component in the complement lectin pathway and binds to Fuc, man, Glu, GlcNAc but not to Gal. Based on the increase in IgG-G0 binding to MBL in vitro; it has been assumed that the MBL pathway of the complement is involved in the pathogenesis of autoimmune diseases. (21)

Also, a high percentage of IgG-GO as well as glucoforms without sialic acid are observed in addition to autoimmune diseases in infections and tumors. (24) We do not know whether the disease itself affects the glycosylation of Ag-specific $\mathrm{Ab}$ in these diseases. There is also a correlation between the percentage of these glycoforms and the activity of the disease. An increase in Gal glycoforms is associated with a remission of the disease in rheumatoid arthritis. The increase in Gal glyforms in pregnancy is associated with the remission of rheumatoid arthritis in pregnant women. (18)

\subsection{Importance of fucose}

The high affinity between Fuc-deficient oligosaccharides (Fuc) and FcyRIIIa is explained as follows: The Fuc residue protrudes into the open space between the Fc segments and prevents close proximity to the oligosaccharide nucleus and inhibits additional auxiliary interactions. Therefore, the lack of Fuc helps these interactions. (25)

Binding to FcyRIIIa, the mediating receptor involved in ADCC is enhanced in afucosyl IgG and FC fragments compared with fucocylated counterparts.(9) The low fucose content results in improved ADCC in vitro using NK cells. Thus, less Abs concentration is required on the surface of the target cell to activate effector cells.

Improved binding to the receptor that translates into improved ADCC is more apparent when the concentration of Abs is reduced. Fucose deficiency requires less $\mathrm{Ab}$ on the surface of the target cell in order for effector cells to function. This is applicable to treatment with monoclonal $\mathrm{Ab}$, which can be administered at lower doses when there is a lack of fucose. (26)

\subsection{Importance of GLCNAC}

B 1,4-acetylglucosaminyltransferase (GnTIII), uses UDP-GlcNAc as a substrate and catalyzes the incorporation of GlcNAc (N-acetylglucosamine) into a $\beta-1.4$ binding to core-mannose in its synthesized oligosaccharide chain with two arms of IgG oligosaccharide, to acquire three bisecting GlcNAc. (27)

It was found that the three-arm digested 
glycoforms showed increased binding to FcyRIIIa and therefore increased ADCC. However, the fucosylated bisecting glycoform has 10 -fold ADCC relative to the fucosylated non-bisecting IgG. (28)

\subsection{Importance of Sialic acid}

Approximately $20 \%$ of human serums in healthy subjects end up with N-acetylneuraminic acid (NANA) which is a sialic acid type. (9) High levels of sialylation have been found to result in low antibody-dependent cytotoxicity (ADCC activity). This is accomplished through reduced binding to the FcyRIIIa of NK cells and is due to the decrease in hinge region flexibility resulting in a reduction in FcyRIIIa binding. The negatively charged and rather diminished sialic acids of the glycan are located close to the hinge region of the IgG.

\section{Clinical Significance of IgG Glycosylation}

\subsection{N-Glucosylation and autoimmunity}

Loss of the FcyRIII activating receptor removes IgG1 effector functions in various disease models such as arthritis, glomerulonephritis, anaphylaxis, immune hemolytic anemia, and immune thrombocytopenia.

The final disease effect depends on the interaction of the activating and inhibitory receptors and, more particularly, on the levels of expression / association of activating / inhibitory receptors. (29)

The abnormal glycan modification accompanies various diseases, such as rheumatoid arthritis, tuberculosis, Crohn's disease, ANCA-associated systemic vasculitis, systemic lupus erythematosus, Sjogren's syndrome and other conditions. An example of a disease that has been studied in relation to the glycan structure is rheumatoid arthritis. (30)

IgG antibodies mediate their activity in addition to, through the fab segment, also via the $\mathrm{N}$-glycosylation of their Fc portion. Increased rates of G0 (no galactose) serum IgG correlate with proinflammatory immune responses as well as the severity of the disease in rheumatoid arthritis and systemic lupus erythematosus. In RA, it appears that G0-IgG antibodies mediate their proinflammatory activity via $\mathrm{Fc} \gamma \mathrm{Rs}$ activating agents as well as via the mannose receptor that is a member of the C-type lectin receptor family.

C-type lectins are proteins and include a large number of transmembrane receptors. Most of these are expressed in dendritic and othermyeloid cells as C-type lectin receptors (CLRs). They recognize carbohydrates and the recognition of glycans by CLRs results in cellular signaling events that regulate the immune response of the cells.
A C-type lectin is Mannose-Binding Lectin (MBL) that is soluble in serum and is the first fraction in the lectin activation pathway. (31)

MBL recognizes the final fucose, glucose, mannose and $\mathrm{N}$-acetylglucosamine, but not galactose. Thus, it is possible to recognize the endpoints exposed to the hepta-saccharide attached at position 297 of the Fc portion of IgG when it is free of galactose. Gal's lack exposes the GlcNac segments of heptasaccharide, which are highly flexible and associated with MBL. High concentrations of GlcNac are required to make the binding, hence high concentrations of IgG. This occurs in autoimmune diseases that have high IgG-G0 concentrations. Thus activation of the complement by MBL leads to chronic inflammation in autoimmune diseases. It is well known that chronic and localized complement activation can cause severe damage to tissues and is an important factor in the pathogenesis of various diseases. (32)

In rheumatoid arthritis, it has been found that there are changes in the N-linked oligosaccharide galactosylation of the Fc portion of serum IgG, with a marked increase in the percentage of IgG lacking galactose (GO form). The oligosaccharides thus terminate in GlcNAc and may expose previously unprotected? Or create new oligosaccharide determinants, which may be immunogenic. IgG-GO levels of glycoforms, correlated with disease activity, decrease in disease remission, as well as during pregnancy, where remission of the disease is observed in patients with RA. (18)

\subsection{Glucosylation of IgG and cancer}

Glycosylation of IgG is of great importance for the functioning of the immune system such as tumor immunoassay through differences that each individual exhibits in its immunological identity and its ability to process and present $\mathrm{Ag}$ in its lymphocytes. Depending on the presence of galactosylation, sialylation and fucosylation of the IgG Fc domain complement activation and dependent cytotoxicity (CDC), Ab-dependent cytotoxicity (ADCC) and anti-inflammatory activity of IgG are affected. All of the above are important for the action of anti-cancer antibodies (anti-Ca-Ab).

From analyzes on IgG glycosylation in various neoplasms, three major changes in glycemic composition were found: 1) reduction of galactosylation; 2) decrease in sialylation; 3) increase in core - fucose in neutral glucan.

The above changes in glycosylation of IgG are observed in gut, ovary, prostate, lung, stomach and multiple myeloma.

It appears that reduced galactosylation (an 
increase in G0-IgG) leads to a higher proinflammatory immune response of $\mathrm{Ab}$, which affects survival by cancer.

But also the reduced sialylation of $\mathrm{Ab}$ (lack of sialic acid on the outer arm of the glycoforms) also leads to a pro-inflammatory reaction and poorer prognosis in cancer patients. (33)

It is known that the development of chronic inflammatory reaction accompanies the development of malignancies.

The increased fucosylation observed in neutral glycans in various types of neoplasms leads to a decrease in ADCC (Ab-dependent cytotoxicity) which is a defense mechanism against cancer cells and therefore promotes the development of cancer. (34) IgG glycosylation is a new prognostic marker for various neoplasms and can classify patients with more aggressive tumors. Changes in glycosylation may be used as biological markers (35) for screening in relation to healthy subjects. It may help in the discovery of patients in early stages of the disease and thus be used as a biological marker to select patients with better prognosis and therefore to use as chemotherapy chemotherapeutic agents with lower toxicity. (32)

\section{Competing Interests}

The authors have declared that no competing interest exists.

\section{References}

1. Edelman GM, Cunningham BA, Gall WE, Gottlieb PD, Rutishauser U, Waxdal MY. The covalent structure of an entire $\gamma \mathrm{G}$ immunoglobulin molecule. The Rockefeller University. Communicated by Theodore Shedlovsky. 1969.

2. Germenis A. Medical Immunology. Papazisis Publications, 2000.

3. Branden C, Tooze J. Introduction to protein structure. 2nd Edition, Garland Publishing, Inc., New York. 1999.

4. Owen JA, Punt J, Stanford SA, Jones PP. KUBY Immunology. Seventh Edition, W. H. Freeman and Company, New York, 2013.

5. Abbas AK, Lichtman AH. Basic Immunology. Functions and Disorders of the Immune System. Saunders, second edition, 2004

6. Schroeder HW Jr, Cavacini L. Structure and Function of Immunoglobulins. J Allergy Clin Immunol, 2009, 125: 41-52

7. Bruhns P. Properties of mouse and human IgG receptors and their contribution to disease models. Blood Journal. 2012; 199: 5640-5649.

8. Biermann MHC, Griffante G, Podolska MJ, Boeltz S, Strurmer J, Munoz LE, Bilyy R, Herrmann M. Sweet but dangerous - the role of immunoglobulin G glycosylation in autoimmunity and imflammation. Lupus. 2016; 25: 934-942.

9. Hmiel LK, Brorson KA, Boyne MT. Post-translational structural modifications of immunoglobulin $\mathrm{G}$ and their effect on biological activity. Anal Bioanal Chem. 2014; Springer.

10. Derry C, Rooperanian DC, Akilesh S. FcRn : The neonatal Fc receptor comes of age. Nature Reviews Immunology. 2007; 7: 715-725.

11. Hirschberg C. Topography of glycosylation in the Rough Endoplasmic Protein Reticulum and Golgi apparatus. Ann. Rev. Biochem. 1987; 56: 63-87.

12. Maverakis E, Kim K, Shimoda M, Gershwin ME, Patel F, Wilken R, Raychaudhuri S, Renee Ruhaak L, Lebrilla CB. Glycans in the immune system and the altered glycan theory of Autoimmunity: A critical review. J Autoiummun. 2015; 0:1-13.

13. Walsh G. Post-translational Modification of Protein Biopharmaceuticals. Part One, Glycosylation. Wiley-VCH Verlag GmbH \& Co. KGaA, Weinheim. 2009.

14. Walsh T. Christopher. Posttranslational Modification of Proteins. Expanding Nature Inventory. Romberts and Company Pumplshers, Englegood, Colorado. 2006

15. Shinkawa T, Nakamura K, Yamane N, Shoji-Hosaka E, Kanda Y, Sakurada M, Uchida K, Anazawa H, Satoh M, Yamasaki M, Hanai N, Shitara K. The absence of fucose but not the presence of Galactose or Bisecting N-Acetylglucosamine of Human IgG1 Complex-type Oligosaccharides shows the critical role of enhancing antibody-dependent cellular cytotoxicity. The journal of biological chemistry. 2003; 278:3466-3473.

16. Arnold JN, Wormald MR, Sim RB, Rudd PM, Dwek RA. Structure of Human Immunoglobulins. Annu. Rev. Immunol. 2007; 25: 21-50.

17. Krapp S, Mimura Y, Jefferis R, Huber R, Sondermann P. Structural Analysis of Human IgG-Fc Glycoforms Reveals a Correlation Between Glycosylation and Structural Integrity. J Mol Biol. 2003; 325: 979-989

18. Ravetch JM, Bolland S. IgG Fc Receptors. Annu. Rev. Immunol. 2001; 19: 275-90.

19. Mimura $\mathrm{Y}$ et al. Contrasting glycosylation profiles between $\mathrm{Fab}$ and $\mathrm{Fc}$ of a human IgG protein studied by electrospray ionization mass spectrometry. Journal of Immunological Methods. 2007; 326: 116-126.

20. Masuda K, Yamaguchi Y, et al. Pairing of oligosaccharides in the Fc region of immunoglobulin G. FEBS Letters. 2000; 473: 349-357.

21. Nimmerjahn F, Anthony RM, Ravetch JV. Agalactosylated IgG antibodies depend on cellular Fc receptors for in vivo activity. Proceedings of the National Academy on Sciences (PNAS). 2007; 104:20.

22. De Haan N, Reiding KR, Driessen G, Van Der Burg M, Wuhrer M. Changes in healthy human IgG Fc-glycosylation after birth and during early childhood. Journal of Proteome Research. 2016; http:/ / pubs.acs.org.

23. Williams PJ, Arkwright PD, Rudd P, Scragg IG, Edge CJ, Wormald MR, Rademacher TW. Short Communication: Selective Placental Transport of Maternal IgG to the Fetus. Placenta. 1995; 16: 749-756.

24. Sazinsky SL, Ott RG, Silver NW, Tidor B, Ravetch JM, Wittrup KD. Aglycosylated immunoglobulin G1 variants productively engage activating Fc receptors. Proceedings of the National Academy on Sciences (PNAS). 2008; 105: 20167-20172

25. Ferrara C, Stuart F, Sondermann P, Brunker P, Umana P. The Carbohydrate at FcyRIIIa Asn-162. An element required for high affinity binding to non-fucosylated IgG glycoforms. The Journal of Biological Chemistry. 2006; 281: 5032-5036.

26. Shields RL, Lai J, Keck R, O'Conneli LY, Hong K, Meng YG, Weikert SHA, Presta L. Lack of Fucose on human IgG1 N-Linked Oligosaccharide improves binding to human FcyRIII and Antibody-dependent Cellular Toxicity. Journal of Biological Chemistry. 2002; 277:26733-26740.

27. Warner TG, deKremer RD, Sjoberg ER, Mock AK. Characterization and analysis of Branched-chain N-Acetylglucosaminyl Oligosaccharides Accumulating in Sandhoff Disease Tissue. The Journal of Biological Chemistry. 1985; 260: 6194-6199.

28. Kibe T, Fujimoto S, Ishida C, Togari H, Wada Y, Okada S, Nakagawa H, Tsukamoto Y, Takahashi N. Glycosylation and Placental Transport of Immunoglobulin G. J Clin Biochem Nutr. 1996; 21:57-63.

29. Nimmerjahn F, Ravetch JV. FcY Receptors: Old Friends and New Family Members. Immunity. 2006; 24: 19-28.

30. Kouings A et al. Site-specific glycosylation of human immunoglobulin G is altered in four rheymatoid arthritis patients. The Biochemical Journal. 1996; 314: 621-630.

31. Malhotra $R$, Wormald MR, Rudd PM, Fischer PB, Dwek RA, Sim RB Glycosylation changes of IgG associated with rheumatoid arthritis can activate complement via the mannose-binding protein. Nature Publishing Group. $1995 ; 1: 237-243$

32. Malhotra R, et al. Glycosylation changes of IgG associated with rheumatoid arthritis can activate complement via the mannose-binding protein. Nature Medicine. 1995; 1: 237-243.

33. Theodoratou E, Thaci K, Agakov F, Timofeeva MN, Stambuk J, Pucic-Bakovic M, Vuckovic F, Orchard P, et al. Glycosylation of plasma IgG in colorectal cancer prognosis. Scientific Reports 6. 2016; article number 28098.

34. Vuckovic F, Theodoratou E, Thaci K, Timofeeva M, Vojta A, Stambuk J, Pucic-Bakovic M, Rudd PM et al. IgG glycome in colorectal cancer. Biology of Human Tumors, Clinical Cancer Research. 2016; American Association for Cancer Research.

35. Ruhaak LR, Miyamoto S, Lebrilla C. Developments in the identification of Glycan Biomarkers for the detection of cancer. Mol Cell Proteomics. 2013; 12: 846-855. 